\title{
BMJ Open Maternal lead exposure and premature rupture of membranes: a birth cohort study in China
}

\author{
Sha Huang, ${ }^{1}$ Wei Xia, ${ }^{1}$ Xia Sheng, ${ }^{1}$ Lin Qiu, ${ }^{1}$ Bin Zhang, ${ }^{2}$ Tian Chen, ${ }^{1}$ Shunqing Xu, ${ }^{1}$ \\ Yuanyuan $\mathrm{Li}^{1}$
}

To cite: Huang S, Xia W, Sheng $X$, et al. Maternal lead exposure and premature rupture of membranes: a birth cohort study in China. BMJ Open 2018;8:e021565. doi:10.1136/ bmjopen-2018-021565

- Prepublication history and additional material for this paper are available online. To view these files, please visit the journal online (http://dx.doi. org/10.1136/bmjopen-2018021565).

\section{SH and WX contributed equally.}

Received 9 January 2018 Revised 9 May 2018 Accepted 11 June 2018

A Check for updates

(c) Author(s) (or their employer(s)) 2018. Re-use permitted under CC BY-NC. No commercial re-use. See rights and permissions. Published by BMJ.

${ }^{1}$ Key Laboratory of Environment and Health (HUST), Ministry of Education \& Ministry of Environmental Protection, and State Key Laboratory of Environmental Health (Incubation), School of Public Health, Tongji Medical College, Huazhong University of Science and Technology, Wuhan, China ${ }^{2}$ Healthcare Department, Wuhan Medical and Health Center for Women and Children, Wuhan, China

Correspondence to

Dr Yuanyuan Li;

liyuanyuan@hust.edu.cn

\section{ABSTRACT}

Objectives Maternal exposure to lead $(\mathrm{Pb})$ has been suggested to correlate with adverse birth outcomes, but evidence supporting an association between $\mathrm{Pb}$ exposure and premature rupture of membranes (PROM) is limited. The aim of our study was to investigate whether maternal $\mathrm{Pb}$ exposure was associated with PROM and preterm PROM.

Design Cross-sectional cohort study.

Study population The present study involved 7290 pregnant women from the Healthy Baby Cohort in Wuhan, China, during 2012-2014.

Main outcome measures PROM was defined as spontaneous rupture of amniotic membranes before the onset of labour and was determined with a $\mathrm{pH} \geq 6.5$ for vaginal fluid. Maternal urinary $\mathrm{Pb}$ level was adjusted by creatinine concentration, and its relationship with PROM was analysed by logistic regression.

Results The IQR of maternal urinary $\mathrm{Pb}$ concentrations of the study population was $2.30-5.64 \mu \mathrm{g} / \mathrm{g}$ creatinine with a median of $3.44 \mu \mathrm{g} / \mathrm{g}$ creatinine. Increased risk of PROM was significantly associated with elevated levels of $\mathrm{Pb}$ in maternal urine (adjusted OR 1.23, 95\% $\mathrm{Cl} 1.0$ to 1.47 for the medium tertile; adjusted OR $1.51,95 \% \mathrm{Cl} 1.27$ to 1.80 for the highest tertile). The risk of preterm PROM associated with $\mathrm{Pb}$ levels was significantly higher when compared with the lowest tertile (adjusted OR 1.24, 95\% C 0.80 to 1.92 for the medium tertile; adjusted OR 1.73, $95 \% \mathrm{Cl} 1.15$ to 2.60 for the highest tertile). In addition, the relationship between $\mathrm{Pb}$ and $\mathrm{PROM}$ was more pronounced among primiparous women than multiparous women ( $p$ for interaction $<0.01$ ).

Conclusions Our study found that higher levels of maternal $\mathrm{Pb}$ exposure was associated with increased risk of PROM, indicating that exposure to $\mathrm{Pb}$ during pregnancy may be an important risk factor for PROM.

\section{INTRODUCTION}

Premature rupture of membranes (PROM) refers to maternal membranes rupture more than 1 hour before the onset of labour, occurs in approximately $5 \%-15 \%$ of deliveries. ${ }^{1-3}$ PROM is related to significant maternal, fetal and neonatal risks, such as maternal infection, prematurity, neonatal sepsis and adverse neurological outcomes. ${ }^{4-6}$ When the

\section{Strengths and limitations of this study}

This study was conducted with a large sample size which included 7290 mother-singleton pairs from a birth cohort study in China.

- All information about the participants was collected from personal interviews and medical records which allowed us to adjust for other potential risk factors for premature rupture of membranes.

- Although many potential confounders were taken into account for analysis, other confounding factors may remain.

rupture occurs prior to 37 weeks of gestation, it is considered as preterm PROM. Preterm PROM appears in 1\%-3\% of all pregnancies and one-third of preterm deliveries, and thus is a leading cause of perinatal morbidity and mortality. ${ }^{57}$ The aetiology of PROM has been shown to be multifactorial, and increasing evidence has regarded exposure to environmental pollutants as risk factors for PROM. ${ }^{8-10}$

Lead $(\mathrm{Pb})$, a ubiquitous non-biodegradable heavy mental that persists in the environment, is widely used in various industries, such as automobiles, paint, batteries and plastics. ${ }^{1112}$ Due to these industrial processes, $\mathrm{Pb}$ has become the most widely distributed toxic heavy metal worldwide. ${ }^{12}$ High levels of $\mathrm{Pb}$ exposure have been demonstrated to be associated with pre-eclampsia, pregnancy-induced hypertension, miscarriage, prematurity, congenital abnormalities and even impaired cognitive function problems in childhood. ${ }^{13-18}$ However, the association between maternal $\mathrm{Pb}$ exposure and risk of PROM is limited, and the results were inconsistent. Some studies have found a significant correlation between the risk of PROM and maternal $\mathrm{Pb}$ levels, ${ }^{10}{ }^{19-21}$ while another study has failed to observe such an association. ${ }^{22}$

China is the largest $\mathrm{Pb}$ (raw and refined) producing and consuming country in the world. ${ }^{23} \mathrm{~Pb}$ pollution poses a significant 
threat to human health, especially for pregnant women and the vulnerable fetuses, who are more susceptible to $\mathrm{Pb}$ exposure since $\mathrm{Pb}$ can freely cross the placenta. ${ }^{24}$ Given this background, the present study involving 7290 participants was designed to explore whether $\mathrm{Pb}$ exposure during pregnancy could increase the risk of PROM and preterm PROM in Chinese pregnant women.

\section{MATERIALS AND METHODS}

\section{Study population and data collection}

The study participants $(n=11311)$ were enrolled between September 2012 and October 2014 from the Healthy Baby Cohort (HBC) study at Wuhan Medical and Health Center for Women and Children in China, and the eligibility criteria have been described previously. ${ }^{25}$ For this study, we excluded women without urine samples $(n=3947)$ and those who delivered infants with congenital malformations $(n=62)$ which may be caused by an abnormal pregnancy. The number of cases with smoking $(n=7)$ or drinking $(n=2)$ during pregnancy were rather small, in line with previous reports, ${ }^{2627}$ and were also excluded, as these lifestyles have been shown to have adverse effects on fetal growth. For women who gave birth twice in HBC $(n=3)$, we excluded the second delivery record and only kept the first one $(n=3)$. Finally, 7290 pregnant women were included in the present study. All participating mothers signed written informed consent at enrolment.

All participants filled out a structural questionnaire after labour during a face-to-face interview by specially trained nurses. Information on the women's demographic and socioeconomic backgrounds (eg, maternal age, educational level and occupational status), prepregnancy body mass index (BMI) (calculated on the basis of self-reported weight and height before pregnancy) and daily-life habits during pregnancy (eg, alcohol and tobacco consumption) were collected during this process. Medical/reproductive histories and outcomes (eg, intrauterine infection, maternal diseases and infant sex) were gathered from medical records. Last menstrual period was used to calculate maternal gestational week.

PROM was defined as spontaneous rupture of amniotic membranes prior to the onset of labour and was determined by the visualisation of amniotic fluid passing from the cervical canal and pooling in the vagina, plus the nitrazine test of a $\mathrm{pH} \geq 6.5$ for vaginal fluid. The nitrazine test is a simple and rapid bedside method to diagnose PROM and is widely used in Chinese hospitals with a relatively high reliability. ${ }^{28}$ The diagnosis of the onset of labour was determined by regular painful contractions and a cervical dilatation of $3 \mathrm{~cm}$ or greater. A rupture that occurred less than 37 weeks of gestation was considered as preterm PROM. The definition of the clinical diagnosis of intrauterine infection was considered in the presence of maternal fever $\left(>38^{\circ} \mathrm{C}\right)$ accompanied by signs or symptoms of maternal and fetal tachycardia, uterine tenderness, foul-smelling discharge, maternal leucocytosis or positive amniotic fluid cultures from an amniocentesis.
Clinical vaginitis was defined by the presence of erythema and an exudative discharge that was associated with symptoms of pruritus or pain. Cervicitis was diagnosed based on cervical erosion with purulent discharge from the cervix. Pelvic inflammatory disease was clinically defined by the presence of adnexal tenderness and/or the presence of tender adnexal mass on bimanual pelvic examination. Vaginal bleeding was defined as the presence of bleeding in pregnant women prior to 28 weeks of gestation. Polyhydramnios was defined by having an amniotic fluid index of $24 \mathrm{~cm}$ or more. Fetal malposition was defined as occipitotransverse or occipitoposterior position.

\section{Urine sample collection and $\mathrm{Pb}$ exposure measurement}

Maternal urine samples were collected on their admission to the hospital while waiting for delivery and were stored immediately in polypropylene tubes at $-20^{\circ} \mathrm{C}$ for further treatment. The detection method for urinary $\mathrm{Pb}$ was introduced previously. ${ }^{29}$ Briefly, prior to analysis, urine specimens were thawed at room temperature. Then, $1 \mathrm{~mL}$ of supernatant urine with $4 \mathrm{~mL}$ of $3 \% \mathrm{HNO}_{3}$ were added into $15 \mathrm{~mL}$ polypropylene tubes for overnight nitrification and were digested by ultrasound for 1 hour at $40^{\circ} \mathrm{C}$. Next, inductively coupled plasma mass spectrometry (ICP-MS; Agilent 7700, Agilent Technologies, Waldbronn) was used to measure maternal urinary $\mathrm{Pb}$ concentrations. Assessment of the instrument performance was conducted using the Standard Reference Material Human Urine (SRM2670a Toxic Elements in Urine, National Institute of Standards and Technology, USA) as external quality control sample in each batch. The concentrations of the quality controls were measured within the certified range recommended by the manufacturer $(5 \%)$. The samples were analysed with an external calibration method using eight standard concentrations ranging from 0 to $500 \mathrm{mg} / \mathrm{L}$. Field and procedure blanks were also included to assess potential contamination, and $\mathrm{Pb}$ was not detected in the containers or storage tubes. The detection rate of maternal urinary $\mathrm{Pb}$ concentrations in this study was $99 \%$, and the samples below the limit of detection (LOD) $(0.01 \mu \mathrm{g} / \mathrm{L})$ were replaced by $1 / 2 \mathrm{LOD}$. The intraday coefficient of variation was below $2.0 \%$, and the interday coefficient of variation was under $3 \%$.

Urine creatinine concentrations measured by an automatic biochemical analyzer (BS-200, Mindray, Shenzhen, China) were used for the adjustment of $\mathrm{Pb}$ concentrations to control variable urine dilutions. The quality control standards for creatinine were identical to those described previously. ${ }^{19}$ The adjusted urinary $\mathrm{Pb}$ concentrations were presented as $\mu \mathrm{g} / \mathrm{g}$ creatinine.

\section{Statistical analyses}

The distribution of $\mathrm{Pb}$ concentration was skewed towards the right when tested by the Kolmogorov-Smirnov normality test. The Wilcoxon signed-rank test was used to compare concentrations of $\mathrm{Pb}$ between PROM and non-PROM women. To evaluate the association between $\mathrm{Pb}$ exposure and $\mathrm{PROM}$, logistic regression analyses 
were conducted to calculate crude and adjusted ORs and 95\% CIs. Maternal urinary $\mathrm{Pb}$ levels were categorised into tertiles, with lowest one used as the reference. We detected the linear trends of $\mathrm{Pb}$ with PROM by modelling the median values of tertiles of $\mathrm{Pb}$ concentration as a continuous variable and used the Wald test to evaluate the statistical significance. The adjustment for potential confounders was based on known factors associated with PROM, such as household income, passive smoking, parity and pregnancy-induced hypertension. ${ }^{4} 70-32$ Additionally, covariates that altered the parameter estimate of $\mathrm{Pb}$ effect on PROM by over $10 \%$ were also included in the final model. Covariates, including maternal age, family income, prepregnancy BMI, parity, passive smoking and pregnancy-induced hypertension, were adjusted for in this analysis. We also performed a sensitivity analysis excluding participants with intrauterine infection (chorioamnionitis), vaginitis, cervicitis, pelvic inflammatory disease, previous vaginal bleeding, polyhydramnios and fetal malposition in consideration of their potential influence on PROM. As data from National Health and Nutrition Examination Survey (NHANES) suggested that women usually have low urinary creatinine, and the upper cut-off $(3 \mathrm{~g} / \mathrm{L})$ remained appropriate for the female population, ${ }^{33}$ a sensitivity analysis that excluded women with creatinine $>3 \mathrm{~g} / \mathrm{L}$ was also conducted. In addition, we analysed the ORs for PROM stratified by maternal parity (primiparous vs multiparous) because the difference in these variables has been previously reported to associate with PROM. ${ }^{32}$ An interaction term was added into the model to assess the effect of $\mathrm{Pb}$ and maternal parity on the outcome of PROM.

All data analyses were performed using SAS V.9.4 (SAS Institute), and two-sided $p$ values below 0.05 were considered statistically significant.

\section{Patient involvement}

No patients were involved in setting the research question or the outcome measures, nor were they involved in the design or implementation of the study. No patients were involved in the interpretation of study results or writing up of the manuscript. There are no plans to disseminate the results of the research to study participants.

\section{RESULTS}

The basic characteristics and urinary $\mathrm{Pb}$ concentrations of 7290 participants is shown in table 1. In this study, the prevalence of PROM and preterm PROM was $12.1 \%$ and $2 \%$, respectively. Maternal age at labour ranged from 18 to 46 years, with 28 years as the average age. Most of the mothers were primiparous $(84.5 \%)$ and had a high educational attainment (>12years) $(67.2 \%)$, high annual family income ( $\geq ¥ 50000$ per year) $(56.9 \%)$ and normal prepregnancy BMI $\left(18.5-23.9 \mathrm{~kg} / \mathrm{m}^{2}\right)(66.3 \%)$. The average gestational age at delivery was 39.2 weeks. About $22.9 \%$ were passively exposed to smoking during pregnancy. Approximately $3.9 \%$ of the pregnant women had hypertension during pregnancy, and $53.4 \%$ of the mothers gave birth to a male infant.

The median of maternal urinary $\mathrm{Pb}$ concentrations in PROM mothers $(3.88 \mu \mathrm{g} / \mathrm{g}$ creatinine) was higher than that of non-PROM mothers (median $=3.39 \mu \mathrm{g} / \mathrm{g}$ creatinine) $(\mathrm{p}<0.05)$. Compared with women without preterm PROM (median $=3.43 \mu \mathrm{g} / \mathrm{g}$ creatinine), the median of maternal urinary $\mathrm{Pb}$ concentrations in preterm PROM mothers was also higher (median $=3.96 \mu \mathrm{g} / \mathrm{g}$ creatinine) $(\mathrm{p}<0.05)$.

Table 2 shows the relationship between creatinine-adjusted maternal urinary $\mathrm{Pb}$ levels and PROM/preterm PROM. Compared with the lowest tertile, a significantly positive correlation between PROM and $\mathrm{Pb}$ concentrations was observed (adjusted OR 1.23, 95\% CI 1.02 to 1.47 for the medium tertile; adjusted OR $1.51,95 \% \mathrm{CI}$ 1.27 to 1.80 for the highest tertile) ( $p$ trend $<0.01$ ). The risk estimate for preterm PROM in association with $\mathrm{Pb}$ levels was significantly higher compared with the lowest tertile (adjusted OR $1.24,95 \%$ CI 0.80 to 1.92 for the medium tertile; adjusted OR $1.73,95 \%$ CI 1.15 to 2.60 for the highest tertile) ( $p$ trend $<0.01$ ). In addition, the sensitivity analysis (see online supplementary material, table S1), excluding subjects with intrauterine infection, vaginitis, cervicitis, pelvic inflammatory disease, previous vaginal bleeding, polyhydramnios and fetal malposition, demonstrated a similar association between $\mathrm{Pb}$ and PROM/preterm PROM. Consistent observation was also made in the sensitivity analysis excluding women with creatinine $>3 \mathrm{~g} / \mathrm{L}$ in the statistical models (see online supplementary material, table S2).

Results stratified by maternal parity are summarised in table 3. Among the 6159 primiparous women, we observed a significantly positive correlation between $\mathrm{Pb}$ concentrations and PROM risk (adjusted OR 1.24, $95 \% \mathrm{CI} 1.03$ to 1.50 for the medium tertile; adjusted OR $1.52,95 \%$ CI 1.27 to 1.83 for the highest tertile) ( $p$ trend $<0.01)$. However, no statistically significant association was found between PROM and $\mathrm{Pb}$ in multiparous mothers (adjusted OR 1.21, 95\% CI 0.65 to 2.25 for the medium tertile; adjusted OR $1.57,95 \%$ CI 0.87 to 2.83 for the highest tertile) ( $p$ trend $=0.13$ ). The risk estimates for PROM in relation to $\mathrm{Pb}$ levels in primiparous and multiparous women were significantly different ( $p$ for interaction $<0.01$ ).

\section{DISCUSSION}

Our study examined the association between maternal urinary $\mathrm{Pb}$ exposure before delivery and risk of PROM in Chinese pregnant women, and we found that urinary $\mathrm{Pb}$ concentration was significantly and positively correlated with PROM and preterm PROM incidence. Meanwhile, our study suggested that the effect of $\mathrm{Pb}$ on PROM may depend on maternal parity, as the correlation is more pronounced in the primiparous women than in the multiparous ones. 
Table 1 Basic characteristics and urinary $\mathrm{Pb}$ concentrations $(\mu \mathrm{g} / \mathrm{g}$ creatinine) of the 7290 pregnant women

\begin{tabular}{|c|c|c|c|}
\hline & & Median (IQR) & \\
\hline Characteristics & N (\%) & $\mathrm{Pb}(\mu \mathrm{g} / \mathrm{g}$ creatinine) & $P$ values \\
\hline Total & 7290 & $3.44(2.30-5.64)$ & \\
\hline Maternal age (years) & & & 0.43 \\
\hline$<25$ & 805 (11.0) & $3.37(2.27-5.52)$ & \\
\hline $25-29$ & $3985(54.7)$ & $3.49(2.32-5.77)$ & \\
\hline $30-34$ & $2011(27.6)$ & $3.40(2.29-5.47)$ & \\
\hline$\geq 35$ & $489(6.7)$ & $3.41(2.15-5.94)$ & \\
\hline Education background (years) & & & 0.63 \\
\hline$\leq 9$ & $1001(13.7)$ & $3.45(2.40-5.63)$ & \\
\hline $9-12$ & $1389(19.1)$ & $3.47(2.35-5.56)$ & \\
\hline$>12$ & $4898(67.2)$ & $3.42(2.26-5.66)$ & \\
\hline Missing & $2(0.03)$ & $5.69(3.50-7.90)$ & \\
\hline Family income(¥/year) & & & 0.37 \\
\hline$<50000$ & $3021(41.4)$ & $3.52(2.31-5.75)$ & \\
\hline$\geq 50000$ & $4148(56.9)$ & $3.39(2.28-5.60)$ & \\
\hline Missing & $121(1.7)$ & $3.50(2.38-5.83)$ & \\
\hline Parity & & & 0.43 \\
\hline primiparous & $6159(84.5)$ & $3.45(2.30-5.66)$ & \\
\hline multiparous & $1131(15.5)$ & $3.38(2.26-5.59)$ & \\
\hline Prepregnancy BMI $\left(\mathrm{kg} / \mathrm{m}^{2}\right)$ & & & 0.10 \\
\hline$<18.5$ & $1527(20.9)$ & $3.47(2.27-5.77)$ & \\
\hline $18.5-23.9$ & $4832(66.3)$ & $3.41(2.29-5.56)$ & \\
\hline$\geq 24$ & $910(12.5)$ & $3.60(2.39-6.04)$ & \\
\hline Missing & $21(0.3)$ & $3.87(2.07-8.12)$ & \\
\hline Passive smoking during pregnancy & & & 0.07 \\
\hline Yes & $1670(22.9)$ & $3.30(2.29-5.32)$ & \\
\hline No & $5620(77.1)$ & $3.47(2.30-5.71)$ & \\
\hline Pregnancy-induced hypertension & & & 0.08 \\
\hline Yes & $286(3.9)$ & $3.26(2.18-5.21)$ & \\
\hline No & $7004(96.1)$ & $3.44(2.30-5.66)$ & \\
\hline Gestational age (weeks) & & & $<0.01$ \\
\hline$<37$ & $291(4.0)$ & $3.99(2.62-7.54)$ & \\
\hline$\geq 37$ & $6999(96.0)$ & $3.42(2.29-5.60)$ & \\
\hline Infant gender & & & 0.02 \\
\hline Male & $3890(53.4)$ & $3.48(2.34-5.78)$ & \\
\hline Female & $3400(46.6)$ & $3.40(2.25-5.52)$ & \\
\hline PROM & $881(12.1)$ & $3.88(2.55-6.59)$ & 0.47 \\
\hline Preterm PROM & $147(2.0)$ & $3.96(2.63-7.67)$ & \\
\hline Term PROM & $734(10.1)$ & $3.87(2.54-6.34)$ & \\
\hline
\end{tabular}

$\mathrm{BMI}$, body mass index; $\mathrm{Pb}$, lead; PROM, premature rupture of membranes.

Preterm PROM is the leading cause of preterm birth and neonatal complications, such as perinatal infections, respiratory distress syndrome, umbilical cord compression, intraventricular haemorrhage, sepsis and even death. ${ }^{57}$ Furthermore, it has been linked with long-term adverse neurodevelopmental outcomes. ${ }^{34}$ Although our current understanding about the cause of PROM is limited, accumulating evidence has suggested that environmental factors play important roles in inducing PROM by stimulating oxidative stress and inflammation. ${ }^{89}$ 


\begin{tabular}{|c|c|c|c|}
\hline $\mathrm{Pb}$ ( $\mu \mathrm{g} / \mathrm{g}$ creatinine) & Case & $\mathrm{OR}^{\star}(95 \% \mathrm{Cl})$ & OR† (95\% Cl) \\
\hline \multicolumn{4}{|l|}{ PROM } \\
\hline Tertile 2 (2.65-4.70) & 291 & 1.24 (1.03 to 1.48$)$ & $1.23(1.02$ to 1.47$)$ \\
\hline Tertile 3 ( $\geq 4.70)$ & 349 & 1.52 (1.27 to 1.81$)$ & 1.51 (1.27 to 1.80$)$ \\
\hline \multicolumn{4}{|l|}{ Preterm PROM } \\
\hline Tertile 1 (<2.65) & 37 & 1.00 & 1.00 \\
\hline Tertile 2 (2.65-4.70) & 46 & 1.25 (0.81 to 1.93$)$ & $1.24(0.80$ to 1.92$)$ \\
\hline Tertile $3(\geq 4.70)$ & 64 & 1.74 (1.16 to 2.62$)$ & $1.73(1.15$ to 2.60$)$ \\
\hline$P$ for trend & & $<0.01$ & $<0.01$ \\
\hline
\end{tabular}

*Unadjusted OR.

†Adjusted for maternal age, family income, pre-BMI, parity, passive smoking and pregnancy-induced hypertension.

$\mathrm{Pb}$, lead; PROM, premature rupture of membranes.

In this study, we observed that maternal $\mathrm{Pb}$ exposure prior to delivery correlated with increased risk of PROM and preterm PROM. After excluding the subjects with complications that are known to cause PROM, or those with creatinine $>3 \mathrm{~g} / \mathrm{L}$, significant associations between $\mathrm{Pb}$ and $\mathrm{PROM} /$ preterm PROM were still observed. Consistent with our findings, a study including 332 pregnant women in Iran reported that one unit increase in the logarithm of maternal blood $\mathrm{Pb}$ concentration was associated with a several-fold increase in the risk of PROM. ${ }^{10}$ Similarly, a study involving 502 pregnant mothers in Columbia found that higher blood levels of $\mathrm{Pb}$ was associated with an increase in the incidence of PROM. ${ }^{19}$ Furthermore, elevated $\mathrm{Pb}$ concentration in the umbilical cord was reported to be associated with increased PROM risk in a cohort study of 749 mother-infant pairs in a Pb-smelter community in South Australia. ${ }^{20}$ Additionally, a study of 89 mother-infant pairs in the Southeast of Spain observed a higher placental $\mathrm{Pb}$ concentration in the PROM cases than in the normal deliveries. ${ }^{21}$ However, despite these findings, an early study demonstrated no significant correlation between PROM risk and blood $\mathrm{Pb}$ concentration measured in 635 samples from the umbilical cord blood. ${ }^{22}$ The reason to this discrepancy is currently unknown, but it may be, at least in part, due to the differences in $\mathrm{Pb}$ exposure levels.

PROM has been reported to be associated with multiple factors, including cigarette smoking, low income, parity, infection and pregnancy-induced hypertension. ${ }^{4} 730-32 \mathrm{In}$ the present study, inclusion of the potential confounding factors for adjustment did not undermine the association between increased levels of $\mathrm{Pb}$ exposure and PROM risk. In the stratified analysis by parity, our results suggested that parity status may influence the effect of maternal $\mathrm{Pb}$ exposure on the risk of PROM. A significantly positive association between PROM and urinary $\mathrm{Pb}$ concentrations was observed in primiparous mothers, whereas a similar positive correlation was also observed in multiparous mothers, although this correlation was not statistically significant. One possible explanation may be the unbalanced sample sizes between primiparous and multiparous mothers (6159 vs 1131). Future studies enrolling more multiparous women will help to further evaluate this difference caused by parity status.

The aetiology and mechanism of the effect of $\mathrm{Pb}$ on PROM are not clear. One prevailing mechanistic explanation is that $\mathrm{Pb}$ can induce toxicity by triggering oxidative stress through the generation of reactive oxygen

Table 3 Risk of PROM associated with the levels of $\mathrm{Pb}$ in maternal urine, stratified by parity

\begin{tabular}{|c|c|c|c|c|c|c|c|}
\hline \multirow{2}{*}{$\begin{array}{l}\text { Pb levels* } \\
\text { ( } \mu \mathrm{g} / \mathrm{g} \text { creatinine) }\end{array}$} & \multicolumn{3}{|c|}{ Primiparous $(n=6159)$} & \multicolumn{3}{|c|}{ Multiparous $(n=1131)$} & \multirow{2}{*}{$\begin{array}{l}P \text { for } \\
\text { interaction }\end{array}$} \\
\hline & $\mathbf{N}$ & OR† (95\% Cl) & OR‡ (95\% Cl) & $\mathbf{N}$ & OR† (95\% Cl) & OR $\ddagger(95 \% \mathrm{Cl})$ & \\
\hline $\mathrm{T} 1$ & 220 & 1.00 & 1.00 & 20 & 1.00 & 1.00 & $<0.01$ \\
\hline T3 & 318 & $1.52(1.27$ to 1.83$)$ & $1.52(1.27$ to 1.83$)$ & 31 & 1.59 (0.89 to 2.84$)$ & 1.57 (0.87 to 2.83$)$ & \\
\hline$P$ for trend & & $<0.01$ & $<0.01$ & & 0.11 & 0.13 & \\
\hline
\end{tabular}

${ }^{*}$ Pb levels: primiparous, T1 (<2.66), T2 (2.66-4.71), T3 ( $\left.\geq 4.71\right)$; multiparous, T1 (<2.61), T2 (2.61-4.61), T3 ( $\left.\geq 4.61\right)$.

†Unadjusted OR.

$\ddagger$ Adjusted for maternal age, family income, pre-BMI, passive smoking and pregnancy-induced hypertension.

$\mathrm{BMI}$, body mass index; $\mathrm{Pb}$, lead; PROM, premature rupture of membranes; $\mathrm{T}$, tertile. 
species ${ }^{1135}$ which is responsible for the structural weakness of collagen fibrils and causes the membranes to lose strength and elasticity, and consequently damage the collagen in fetal membrane. ${ }^{10} 36{ }^{37}$ Furthermore, $\mathrm{Pb}$ is shown to induce inflammatory responses via upregulating the expression of proinflammatory cytokines, such as tumor necrosis factor (TNF)- $\alpha,{ }^{38}$ thus predispose the membrane to rupture by promoting alterations of membrane fluidity and impairment in membrane barrier function. ${ }^{39}$

Urinary $\mathrm{Pb}$ is favoured for long-term biomonitoring and is widely used in the assessment of $\mathrm{Pb}$ exposure level. ${ }^{404}$ In the present study, maternal urinary $\mathrm{Pb}$ concentration (arithmetic mean $(\mathrm{AM})=7.40 \mu \mathrm{g} / \mathrm{g}$ creatinine, geometric mean $(\mathrm{GM})=3.69 \mu \mathrm{g} / \mathrm{g}$ creatinine, median $=3.44 \mu \mathrm{g} / \mathrm{g}$ creatinine) was higher than pregnant mothers reported in several developed countries, such as Australia $(\mathrm{AM}=0.87 \mu \mathrm{g} / \mathrm{g}$ creatinine, median $=0.7 \mu \mathrm{g} / \mathrm{g}$ creatinine) ${ }^{42}$ Japan (GM=0.48 $\mu \mathrm{g} / \mathrm{g}$ creatinine $)^{43}$ and the USA $(\mathrm{GM}=0.63 \mu \mathrm{g} / \mathrm{L}) .{ }^{44}$ Yet, the urinary $\mathrm{Pb}$ concentration in our study also overlapped with other countries, including Spain $(\mathrm{AM}=5.2 \mu \mathrm{g} / \mathrm{g}$ creatinine, median $=3.9 \mu \mathrm{g} / \mathrm{g}$ creatinine).$^{45}$ In addition, $\mathrm{Pb}$ concentration in the present subjects was generally lower in comparison with pregnant women reported in low-income and middle-income countries, such as Nigeria (AM=28.5 $\mu \mathrm{g} / \mathrm{g}$ creatinine) ${ }^{46}$ Although $\mathrm{Pb}$-containing petrol has been phased out since 2000 in China, $\mathrm{Pb}$ pollution remains a huge environmental challenge, as large amounts of $\mathrm{Pb}$ pollutants from various sources of $\mathrm{Pb}$ consumption have been increasing rapidly due to the unprecedented economic development. ${ }^{47} 48$ In the past decades, elevated accumulations of $\mathrm{Pb}$ have been widely spread in soil and dust in many Chinese provinces ${ }^{23}{ }^{48}$ raising a major public health concern in China, since $\mathrm{Pb}$ can enter human body via intake of Pb-containing food, water and even air. ${ }^{12}{ }^{48} \mathrm{As}$ a consequence, excessive $\mathrm{Pb}$ emissions pose serious adverse health effects on humans, especially on the susceptible pregnant women and their fetuses.

The strength of this study is as follows: first, it was conducted with a large sample size which included 7290 mother-singleton pairs from a birth cohort study in China. We performed sensitivity analyses and stratified analyses to evaluate the relationship between maternal $\mathrm{Pb}$ exposure and $\mathrm{PROM}$, where a significant correlation was observed. Moreover, all information about the participants including demographic characteristics, socioeconomic status and pregnancy outcomes were gathered from personal interviews and medical records which made it possible to adjust for other potential risk factors for PROM.

Admittedly, there were other potential confounders that we were not able to control. Unfortunately, several important risk factors for PROM, such as drug use, cervical insufficiency and premature contractions, were not collected in the present study but will be included in the future studies. The small numbers of preterm PROM and multiparous women also limited the power of our study. In addition, urinary $\mathrm{Pb}$ collected and measured at labour only reflects plasma $\mathrm{Pb}$ level at labour which may not accurately reflect the dynamic maternal $\mathrm{Pb}$ exposure throughout the whole pregnancy and limit the strength to determine the causal effect between maternal urinary $\mathrm{Pb}$ level and PROM. Therefore, further studies with urine samples collected at multiple time points and from different populations are needed to confirm the observed relationship between $\mathrm{Pb}$ and PROM.

\section{CONCLUSION}

In this study, we observed a positive relationship between maternal urinary $\mathrm{Pb}$ and the risk of PROM, supporting that maternal exposure to $\mathrm{Pb}$ may be a potential risk factor for PROM. Additionally, the significant association was only present in primiparous women and not in multiparous women. This finding suggested that appropriate public health measures need to be implemented to control maternal $\mathrm{Pb}$ exposure during pregnancy.

Contributors SH carried out the statistical analyses and drafted the manuscript. WX and XS assisted in the statistical analyses, critically reviewed and revised the manuscript. LQ, BZ, TC and SX contributed to the study design and developed the initial protocol. YL contributed to the study design, critically reviewed and revised the manuscript. All authors read and approved the final manuscript.

Funding This work was supported by the National Natural Science Foundation of China $(91743103,91643207,21437002,81372959$, and 81402649), the National Key Research and Development Plan (2016YFC0206700, 2016YFC0206203), and the Fundamental Research Funds for the Central Universities HUST (2016YXZD043, 2015ZDTD047).

\section{Competing interests None declared.}

Patient consent Not required.

Ethics approval The ethical committees of Tongji Medical College, Huazhong University of Science and Technology and the study hospital approved the study protocol.

Provenance and peer review Not commissioned; externally peer reviewed.

Data sharing statement Extra data are available by emailing to the corresponding author at liyuanyuan@hust.edu.cn.

Open access This is an open access article distributed in accordance with the Creative Commons Attribution Non Commercial (CC BY-NC 4.0) license, which permits others to distribute, remix, adapt, build upon this work non-commercially, and license their derivative works on different terms, provided the original work is properly cited, appropriate credit is given, any changes made indicated, and the use is non-commercial. See: http://creativecommons.org/licenses/by-nc/4.0/.

\section{REFERENCES}

1. Ural SH, Nagey DA. Premature Rupture of Membranes. Postgraduate Obstetrics \& Gynecology 1998;18:1-3.

2. ACOG Committee on Practice Bulletins-Obstetrics. ACOG Practice Bulletin No. 80: premature rupture of membranes. Clinical management guidelines for obstetrician-gynecologists. Obstet Gynecol 2007;109:1007-19.

3. Andres RL, Day MC. Perinatal complications associated with maternal tobacco use. Semin Neonatol 2000;5:231 41.

4. Poma PA. Premature rupture of membranes. J Natl Med Assoc 1996;88:27.

5. Mercer BM. Preterm premature rupture of the membranes. Obstet Gynecol 2003;101:178-93.

6. Tchirikov M, Schlabritz-Loutsevitch N, Maher J, et al. Mid-trimester preterm premature rupture of membranes (PPROM): etiology, diagnosis, classification, international recommendations of treatment options and outcome. J Perinat Med 2017. 
7. Simhan HN, Canavan TP. Preterm premature rupture of membranes: diagnosis, evaluation and management strategies. BJOG 2005;112:32-7.

8. Wallace ME, Grantz KL, Liu D, et al. Exposure to ambient air pollution and premature rupture of membranes. Am J Epidemiol 2016;183:1114-21.

9. Dadvand P, Basagaña X, Figueras F, et al. Air pollution and preterm premature rupture of membranes: a spatiotemporal analysis. $A m \mathrm{~J}$ Epidemiol 2014;179:200-7.

10. Vigeh M, Yokoyama K, Shinohara A, et al. Early pregnancy blood lead levels and the risk of premature rupture of the membranes. Reprod Toxicol 2010;30:477-80.

11. Flora G, Gupta D, Tiwari A. Toxicity of lead: a review with recent updates. Interdiscip Toxicol 2012;5:47-58.

12. Cheng $\mathrm{H}, \mathrm{Hu} \mathrm{Y}$. Lead $(\mathrm{Pb})$ isotopic fingerprinting and its applications in lead pollution studies in China: a review. Environ Pollut 2010;158:1134-46.

13. Vigeh M, Yokoyama K, Ramezanzadeh F, et al. Lead and other trace metals in preeclampsia: a case-control study in Tehran, Iran. Environ Res 2006;100:268-75.

14. Chen XK, Yang Q, Smith G, et al. Environmental lead level and pregnancy-induced hypertension. Environ Res 2006;100:424-30.

15. Needleman HL, Rabinowitz M, Leviton A, et al. The relationship between prenatal exposure to lead and congenital anomalies. JAMA 1984;251:2956-9.

16. Dietrich KN, Succop PA, Berger OG, et al. Lead exposure and the cognitive development of urban preschool children: the Cincinnati Lead Study cohort at age 4 years. Neurotoxicol Teratol 1991;13:203-11.

17. Hertz-Picciotto I. The evidence that lead increases the risk for spontaneous abortion. Am J Ind Med 2000;38:300-9.

18. Vigeh M, Yokoyama K, Seyedaghamiri Z, et al. Blood lead at currently acceptable levels may cause preterm labour. Occup Environ Med 2011;68:231-4.

19. Fahim MS, Fahim Z, Hall DG. Effects of subtoxic lead levels on pregnant women in the state of Missouri. Res Commun Chem Pathol Pharmacol 1976;13:309-31.

20. Baghurst PA, Robertson EF, Oldfield RK, et al. Lead in the placenta, membranes, and umbilical cord in relation to pregnancy outcome in a lead-smelter community. Environ Health Perspect 1991;90:315-20.

21. Falcón $M$, Viñas $P$, Luna A. Placental lead and outcome of pregnancy. Toxicology 2003;185(1-2):59-66.

22. Angell NF, Lavery JP. The relationship of blood lead levels to obstetric outcome. Am J Obstet Gynecol 1982;142:40-6.

23. Chen HY, Li AJ, Finlow DE. The lead and lead-acid battery industries during 2002 and 2007 in China. J Power Sources 2009;191:22-7.

24. Shannon M. Severe lead poisoning in pregnancy. Ambul Pediatr 2003;3:37-9.

25. Yang J, Huo W, Zhang B, et al. Maternal urinary cadmium concentrations in relation to preterm birth in the Healthy Baby Cohort Study in China. Environ Int 2016;94:300-6.

26. West R. Tobacco control: present and future. Br Med Bull 2006;7778:123-36.

27. Cochrane J, Chen $\mathrm{H}$, Conigrave KM, et al. Alcohol use in China. Alcohol Alcohol 2003;38:537-42.

28. Liang DK, Qi HB, Luo X, et al. Comparative study of placental $\alpha$-microglobulin-1, insulin-like growth factor binding protein-1 and nitrazine test to diagnose premature rupture of membranes: a randomized controlled trial. J Obstet Gynaecol Res 2014;40:1555-60.

29. Xia W, Du X, Zheng T, et al. A case-control study of prenatal thallium exposure and low birth weight in China. Environ Health Perspect 2016;124:164-9.
30. Lee T, Silver H. Etiology and epidemiology of preterm premature rupture of the membranes. Clin Perinatol 2001;28:721-34.

31. Zhou Q, Zhang W, Xu H, et al. Risk factors for preterm premature rupture of membranes in Chinese women from urban cities. Int $J$ Gynaecol Obstet 2014;127:254-9.

32. Naeye RL. Factors that predispose to premature rupture of the fetal membranes. Obstet Gynecol 1982;60:93-8.

33. Barr DB, Wilder LC, Caudill SP, et al. Urinary creatinine concentrations in the U.S. population: implications for urinary biologic monitoring measurements. Environ Health Perspect 2005;113:192-200.

34. Clark EA, Varner M. Impact of preterm PROM and its complications on long-term infant outcomes. Clin Obstet Gynecol 2011;54:358-69.

35. Hsu PC, Liu MY, Hsu CC, et al. Lead exposure causes generation of reactive oxygen species and functional impairment in rat sperm. Toxicology 1997;122(1-2):133-43.

36. Moore RM, Mansour JM, Redline RW, et al. The physiology of fetal membrane rupture: insight gained from the determination of physical properties. Placenta 2006;27(11-12):1037-51.

37. Wall PD, Pressman EK, Woods JR. Preterm premature rupture of the membranes and antioxidants: the free radical connection. $J$ Perinat Med 2002;30:447-57.

38. Ghareeb DA, Hussien HM, Khalil AA, et al. Toxic effects of lead exposure on the brain of rats: Involvement of oxidative stress, inflammation, acetylcholinesterase, and the beneficial role of flaxseed extract. Toxicological \& Environmental Chemistry 2010;92:187-95.

39. Gervasi MT, Chaiworapongsa T, Naccasha N, et al. Maternal intravascular inflammation in preterm premature rupture of membranes. J Matern Fetal Neonatal Med 2002;11:171-5.

40. Barbosa F, Tanus-Santos JE, Gerlach RF, et al. A critical review of biomarkers used for monitoring human exposure to lead: advantages, limitations, and future needs. Environ Health Perspect 2005;113:1669-74.

41. Abadin H, Ashizawa A, Stevens YW, et al. Agency for Toxic Substances and Disease Registry (ATSDR) Toxicological Profiles. Toxicological Profile for Lead. Atlanta (GA): Agency for Toxic Substances and Disease Registry (US), 2007.

42. Hinwood AL, Callan AC, Ramalingam M, et al. Cadmium, lead and mercury exposure in non smoking pregnant women. Environ Res 2013;126:118-24.

43. Shirai S, Suzuki Y, Yoshinaga J, et al. Maternal exposure to low-level heavy metals during pregnancy and birth size. J Environ Sci Health $A$ Tox Hazard Subst Environ Eng 2010;45:1468-74.

44. Jain RB. Effect of Pregnancy on the Levels of Urinary Metals for Females Aged 17-39 Years Old: Data From National Health and Nutrition Examination Survey 2003-2010. J Toxicol Environ Health A 2013;76:86-97.

45. Fort M, Cosín-Tomás M, Grimalt JO, et al. Assessment of exposure to trace metals in a cohort of pregnant women from an urban center by urine analysis in the first and third trimesters of pregnancy. Environ Sci Pollut Res Int 2014;21:9234-41.

46. Adekunle IM, Ogundele JA, Oguntoke O, et al. Assessment of blood and urine lead levels of some pregnant women residing in Lagos, Nigeria. Environ Monit Assess 2010;170:467-74.

47. Yan $\mathrm{CH}, \mathrm{Xu}$ J, Shen XM. Childhood lead poisoning in China: challenges and opportunities. Environ Health Perspect 2013;121:A29 4-A295.

48. Duzgoren-Aydin NS. Sources and characteristics of lead pollution in the urban environment of Guangzhou. Sci Total Environ 2007;385:182-95. 